\title{
SIMPLIFIED ANALTIC FORMULAE FOR MAGNETO-OPTICAL KERR EFFECTS IN GENERAL CASES
}

\author{
Chun-Yeol You and Sung-Chul Shin \\ Department of Physics, Korea Advanced Institute of Science and Technology, \\ Taejon 305-701, Korea
}

\section{Introduction}

Recently, the magneto-optical Kerr effects(MOKE) have been attracting much attention due to applications of the effect as a tool in probing magnetism as well as a readout mechanism in highdensity magneto-optical recording. Furthermore, magnetic thin films have been attracting much attention due to a wealth of interesting physical properties and their applications. To understand their novel magnetic properties, the MOKE has been widely used because of its high sensitivity to the spinpolarized electronic band structure which causes magnetism. The quantitative analysis of the MOKE for the special and simple cases, where the incident angle of light is normal and the direction of the magnetization is in-plane or perpendicular to the film plane, has been widely studied by many researchers. The quantitative analysis of the MOKE signal on the general cases, where the direction of the magnetization is arbitrary and the direction of the incident beam is not normal, is quite important for the wide applications of the MOKE methods to investigate magnetic thin films. However, it is not simple because of their complicated relations[1-3]. Due to their complicated relations, the studies about the MOKE have been limited by their analytic analyses. However, quite recently[4], we have derived simplified analytic formulae for the MOKE of an optically thick magnetic film. In this study, we extend these formulae for the ultrathin magnetic films which exhibit a novel interesting physical properties.

\section{Simplified Analytic Formulae for MOKE in General Cases}

There are various kinds of MOKE depending on the relative direction of the magnetization to the plane of incidence. Depending on the direction of the magnetization whether it is parallel to the surface normal, parallel to the surface and in the plane of incidence, or parallel to the surface and perpendicular to the plane of incidence, it is called the polar Kerr effect, the longitudinal Kerr effect, and the transverse (or equatorial) Kerr effect, respectively.

In Fig. 1, a beam of light passes from a non-magnetic medium 0 to another non-magnetic medium 2 through a magnetic medium 1 having an arbitrary direction of the magnetization and thickness $d_{1}$. When $d_{1}$ is very thick $\left(2 \pi\left|\eta_{1}\right| d_{1} \gg \lambda\right)$, we can ignore the multiple reflections. In this case, we can simplify the expressions for the MOKE as follows using Snell's law and simple trigonometric relations [4]:

$$
\begin{aligned}
& \Theta_{K}^{p} \equiv \theta_{K}^{p}+i \varepsilon_{K}^{p} \equiv \frac{r_{s p}}{r_{p p}}=\frac{\cos \theta_{0}\left(m_{z}+m_{y} \tan \theta_{1}\right)}{\cos \left(\theta_{0}+\theta_{1}\right)} \Theta_{n}, \\
& \Theta_{K}^{s} \equiv \theta_{K}^{s}+i \varepsilon_{K}^{s} \equiv \frac{r_{p s}}{r_{s s}}=\frac{\cos \theta_{0}\left(m_{z}-m_{y} \tan \theta_{1}\right)}{\cos \left(\theta_{0}-\theta_{1}\right)} \Theta_{n} .
\end{aligned}
$$

Here, $\Theta_{n}=\frac{i n_{0} n_{1} Q}{\left(n_{1}^{2}-n_{0}^{2}\right)}$ is the well-known complex polar Kerr effect for normal incidence. $Q$ is magneto-optical constant defined $i \varepsilon_{x y} / \varepsilon_{x x}$, and $r_{i j}$ are the ratio of the incident $j$ polarized field and reflected $i$ polarized electric field. The $n_{j}$ and $\theta_{j}$ are complex refractive indices and refractive angle for $j$ -

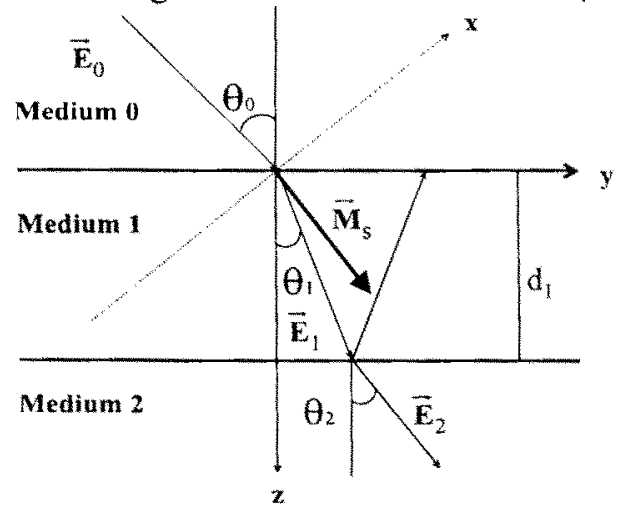

Fig. 1 The coordinate system of the nonmagnetic medium 0 and 2 , and the magnetic medium 1 . The magnetization direction of the medium 1 is arbitrary. 
th layer, and $m_{i}$ are the direction cosines of the magnetization vector. The superscript $p$ and $s$ mean the $p$ - and $s$-polarized waves. The only difference between $p$ - and $s$-polarized waves is the sign of the argument of the cosine function in the denominator of the prefactors. For the ultrathin limit case, $\left(2 \pi|n| d_{1} \ll \lambda\right)$ multiple reflections should be considered. Using the medium propagation matrices and medium boundary matrices[2], we can obtained the following relations in the first-order approximations of the $\frac{2 \pi\left|n_{1}\right| d_{1}}{\lambda}$ and $Q$ :

$$
\begin{aligned}
& \Theta_{K}^{p} \equiv \frac{r_{p p}}{r_{p p}}=\frac{\cos \theta_{0}}{\cos \left(\theta_{0}+\theta_{2}\right)}\left(m_{z} \cos \theta_{2}+m_{y} \frac{\sin ^{2} \theta_{1}}{\sin \theta_{2}}\right) \Theta_{n}^{0}, \\
& \Theta_{K}^{s} \equiv \frac{r_{p s}}{r_{s s}}=\frac{\cos \theta_{0}}{\cos \left(\theta_{0}-\theta_{2}\right.}\left(m_{z} \cos \theta_{2}-m_{y} \frac{\sin ^{2} \theta_{1}}{\sin \theta_{2}}\right) \Theta_{n}^{0} .
\end{aligned}
$$

Here, $\Theta_{n}^{0} \equiv \frac{4 \pi n_{0} d_{1} n_{1}^{2} Q}{\lambda\left(n_{s}^{2}-n_{0}^{2}\right)}$ is the complex polar Kerr effects for normal incidence for ultrathin magnetic medium 1. For the ultrathin limit case, the refractive angle of the substrate $\theta_{2}$ would appear instead of that of the magnetic medium 1 in the prefactors.

\section{Comparison to the Experimental Data}

We have applied these results to the published experimental data on $\mathrm{Co} / \mathrm{Pd}$ and $\mathrm{Cu} / \mathrm{Co}$ multilayers by Deeter[5], where he has measured the polar Kerr rotation angles of $(1.8-\AA \quad \mathrm{Co} / 9-\AA \mathrm{Pt})_{200}$ multilayer and the longitudinal Kerr rotation angles of $(50-\AA$ $\mathrm{Cu} / 55.8-\AA \mathrm{Co})_{10}$ multilayer for $p$ and $s$ - polarizations. The Kerr rotation angles of $p$ - and $s$ polarized waves were reported at wide incident angles ranging from $5^{\circ}$ to $85^{\circ}$ with an increment of $5^{\circ}$.
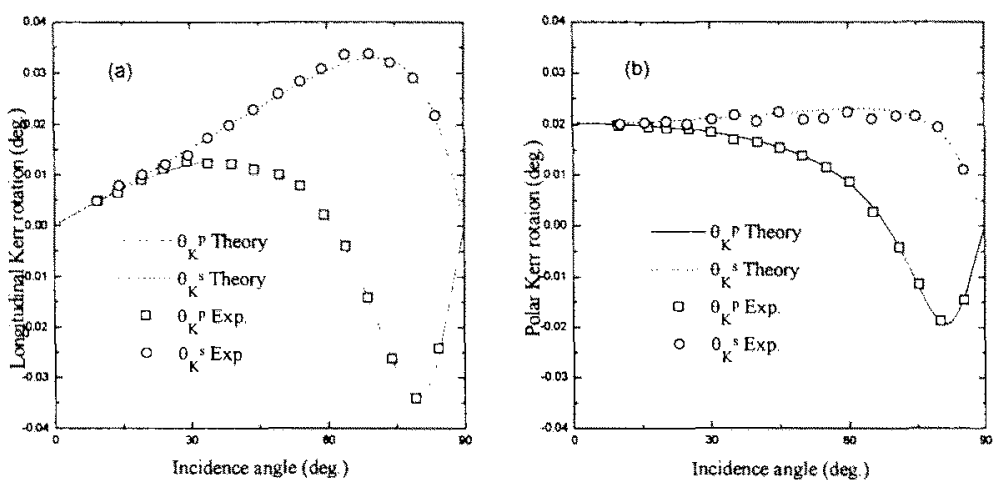

Fig. 2 Experimental and theoretical p- and s- Kerr rotation curves of (a) the $\mathrm{Cu} / \mathrm{Co}$ and (b) the $\mathrm{Co} / \mathrm{Pd}$ multilayers.

The calculated results using present simplified analytic formulae with the experimental data are shown in Fig. 2. As seen in the Fig. 2, the experimental data are well explained by the present simplified formulae.

\section{Conclusions}

We have derived simplified analytic formulae for the MOKE of optically thick and optically ultrathin cases in general configurations. We found that they could be described as product of two factors whether optically thick or ultrathin: prefactors are function of the optical parameters of the system and the main factors are polar Kerr effects for each case. The formulae will be useful to study the MOKE of a magnetic sample having arbitrary magnetizatioin.

\section{References}

[1] R. P. Hunt, J. Appl. Phys. 38, 1652 (1967).

[2] J. Zak, E. R. Moog, C. Liu, and S. D. Bader, J. Appl. Phys. 68, 4203 (1990).

[3] Y. J. Yang and M. R. Scheinfein, J. Appl. Phys. 74, 6810 (1993).

[4] C.-Y. You and S.-C. Shin, Appl. Phys. Lett. 69, 1315 (1996).

[5] M. N. Deeter and D. Sarid, IEEE. Trans. Magn. MAG-24, 2470 (1988). 